\title{
Midterm results of extensive primary repair of the thoracic aorta by means of total arch replacement with open stent graft placement for an acute type $A$ aortic dissection
}

\author{
Naomichi Uchida, MD, Hiroshi Ishihara, MD, Hidenori Shibamura, MD, Yoshiki Kyo, MD, and Masamiti Ozawa, MD
}

See related editorial on page 777 .
From the Division of Cardiovascular Surgery, Hiroshima-city Asa General Hospital, Hiroshima, Japan.

Received for publication May 20, 2005; revisions received Aug 24, 2005; accepted for publication Aug 30, 2005.

Address for reprints: Naomichi Uchida, MD, Division of Cardiovascular Surgery, Hiroshima-city Asa General Hospital, 2-1-1, Kabe-minami, Asa-Kita-Ku, Hiroshima, Japan, 731-0293 (E-mail: n-utida@ asa-hosp.city.hiroshima.jp)

J Thorac Cardiovasc Surg 2006;131:862-7

$0022-5223 / \$ 32.00$

Copyright $\odot 2006$ by The American Association for Thoracic Surgery

doi:10.1016/j.jtcvs.2005.08.061
Objectives: We sought to describe the midterm results of extensive primary repair of the thoracic aorta by means of the modified elephant trunk technique with a stent graft for acute type A aortic dissection, particularly the changes of the false lumen shown by enhanced computed tomographic scanning.

Methods: The subjects were 35 consecutive patients who received arch replacement with open stent grafting for type A acute aortic dissection between December 1997 and April 2002. The mean follow-up period was 55 months (range, 30-83 months). Computed tomographic scanning was performed at $1,3,12$, and 36 months postoperatively to detect thrombosis and obliteration of the false lumen after its exclusion by the stent graft. The diameter of the aorta was measured at 3 levels: the distal edge of the stent graft, the diaphragm, and the origin of the superior mesenteric artery.

Results: Two patients died in the initial operation, but no patients required additional surgical treatment of the thoracic aorta. The mean diameter of the stent grafts was 26.2 $\mathrm{mm}$, and the mean length was $8.9 \mathrm{~cm}$. Thrombus formation in the false lumen was recognized at the distal edge of the graft in all patients, at the diaphragmatic level in 26 patients, and at the superior mesenteric artery level in 15 patients. Obliteration of the false lumen was recognized at the distal edge of the graft in all patients, at the diaphragmatic level in 20 patients, and at the superior mesenteric artery level in 15 patients. The aorta distal to the stent graft showed minimal changes.

Conclusions: In patients with acute type A aortic dissections, it is possible to perform extensive primary repair of the thoracic aorta with relative safety by using a synthetic graft with a self-expanding stent, and this method might reduce the necessity of further operations not only for the distal descending aorta but also for the thoracoabdominal aorta.

A cute type A aortic dissection frequently requires emergency surgical treatment. Conventional replacement is often limited to the region from the ascending aorta to the aortic arch, ${ }^{1}$ and therefore a false lumen sometimes remains distal to the descending thoracic aorta after surgical intervention that can significantly influence the early and late prognosis. ${ }^{2}$ We previously reported good early results after treating type A acute aortic dissection by means of extensive primary repair of the thoracic aorta by using the modified elephant trunk technique with a stent graft (so-called open stent graft placement). ${ }^{3}$ This article describes the midterm results of this procedure, particularly the fate of the false lumen, as shown by means of enhanced computed tomographic (CT) scanning.

\section{Methods}

\section{Subjects}

We performed emergency total arch replacement by means of transaortic stent grafting (open stent grafting) in 35 consecutive patients with type A acute aortic dissection within 48 hours 


\section{Abbreviations and Acronyms \\ $\mathrm{CT}=$ computed tomography \\ $\mathrm{SMA}=$ superior mesenteric artery}

from the onset of dissection between December 1997 and April 2002 performed by 2 surgeons (Drs Ishihara and Uchida). The patients consisted of 13 men and 22 women aged from 47 to 80 years, with a median age of 67.8 years. The operative indications for open stent grafting to repair type A aortic dissection were an age of less than 80 years and the extension of the false lumen from the ascending aorta to the abdominal aorta, with the primary tear anywhere in that region. The operative procedure has been described in a previous report by Ishihara and colleagues. ${ }^{3}$ The size and length of the stent graft were determined by means of intraoperative measurement with a ball-shaped sizer inserted into the true lumen of the descending aorta through a transverse incision under transesophageal echocardiographic guidance. The distal edge of the stent graft was positioned at the level of the main pulmonary trunk. The primary tear was distal to the left subclavian artery (DeBakey subtype III-D) in 6 patients, and the primary tears were covered by the stent graft.

\section{Operative Method}

A median sternotomy was performed after achievement of general anesthesia. An arterial perfusion cannula was inserted into the femoral artery, the right axillary artery, or both; venous drainage cannulas were inserted into the superior and inferior venae cavae from the right atrium; and total extracorporeal circulation was commenced. Hypothermic circulation was used to reduce the rectal temperature to $25^{\circ} \mathrm{C}$. A left ventricular vent tube was inserted from the right superior pulmonary vein, and aspiration was performed. A crossclamp was applied to the peripheral part of the ascending aorta, a small incision was made in the right atrium, and retrograde cardioplegia was performed under direct vision. The ascending aorta was dissected transversely at the proximal end. Gelatinresorcin-formol glue (Cardial, Tecnopole) was injected into the false lumen on the proximal side of the ascending aorta, and then the false lumen was dosed. When it was confirmed that the rectal temperature had decreased to $25^{\circ} \mathrm{C}$, total circulatory arrest was achieved. The clamp was removed, and the aortic arch was incised longitudinally until immediately before the origin of the left subclavian artery. At the end point, the aortic arch was dissected transversely. Three balloon catheters were inserted into the brachiocephalic artery, the left common carotid artery, and the left subclavian artery for perfusion at a rate of 300, 200, and 100 $\mathrm{mL} / \mathrm{min}$, respectively, to maintain perfusion of the brain. A ballshaped sizer was inserted into the true lumen of the descending thoracic aorta from the transverse incision of the aortic arch, and then the exact diameter of the true lumen was measured. A synthetic graft 7 to $12 \mathrm{~cm}$ long, which was previously attached by means of a self-expandable Z-shaped stent (William Cook Europe $\mathrm{A} / \mathrm{S}$ ) with the tip $5 \mathrm{~cm}$ on the distal side, was selected with a diameter 1 to $3 \mathrm{~mm}$ larger than that measured. Then the distally stented graft was placed in a $30 \mathrm{~F}$ introducer, which was inserted into the descending aorta according to the method reported by
Kato and colleagues. ${ }^{4}$ The graft was fixed in the true lumen of the descending aorta by means of expansion of the Z-shaped stent and aortic blood pressure. The graft was pulled to the transverse dissection line in the distal aortic arch and trimmed to match the dissection line. The left subclavian artery was dissected transversely at the proximal end, and the proximal stump was closed with 4-0 polypropylene sutures. The adventitia of the aortic stump was covered with a felt strip $2 \mathrm{~cm}$ wide, and the stump was reinforced with continuous 4-0 polypropylene sutures. A synthetic graft with 4 branches was anastomosed end to end to the stump of the distal aortic arch with continuous 3-0 polypropylene sutures. Then the third branch was anastomosed to the left subclavian artery. The proximal graft was crossclamped, antegrade systemic perfusion from the fourth branch was started, and the patient was rewarmed by means of extracorporeal circulation. Next, the proximal graft was anastomosed to the stump of the ascending aorta, and coronary perfusion was started. The left common carotid artery and the brachiocephalic artery were anastomosed to respective branches of the graft in succession. This completed the procedure (Figure 1, A). The mean duration of extracorporeal circulation with selective cerebral perfusion, mean total extracorporeal
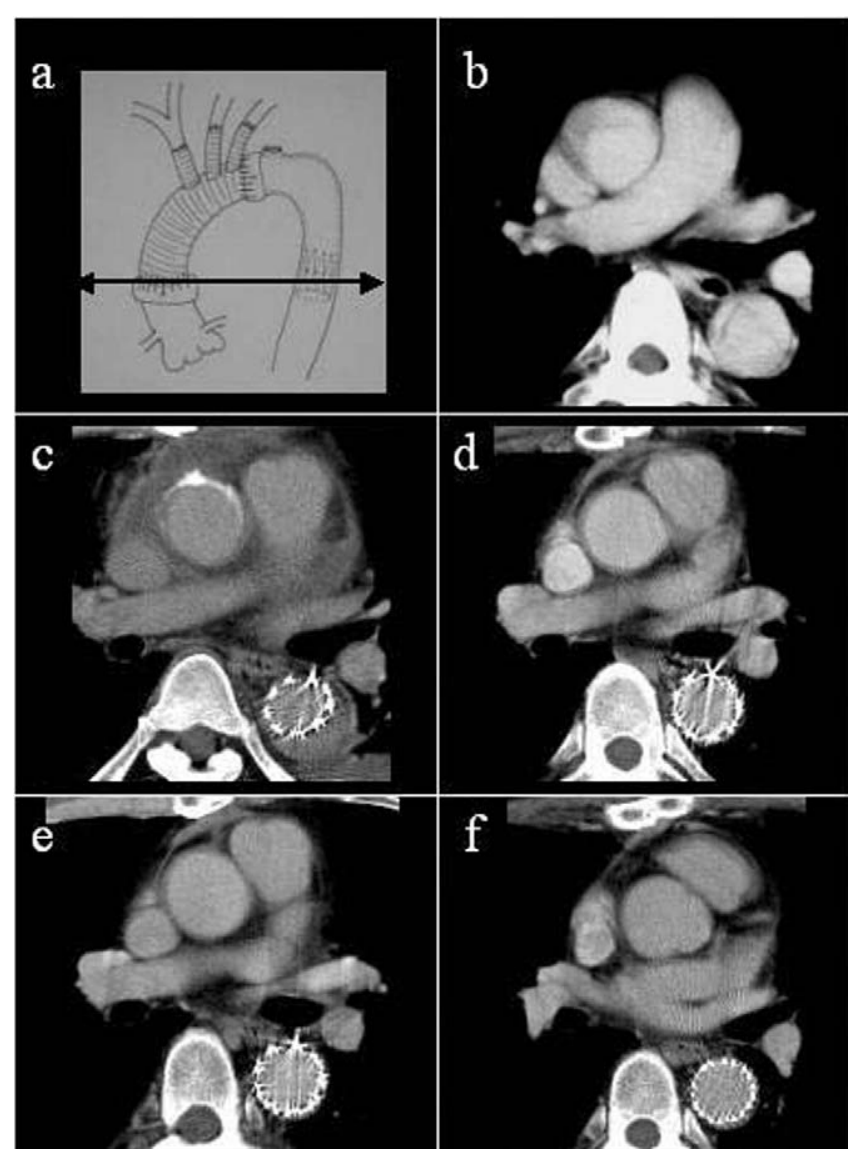

Figure 1. Postoperative CT follow-up study. A, Level examined: edge of the stent graft. B, Preoperative findings. C, One month postoperatively. D, Three months postoperatively. E, One year postoperatively. F, Three years postoperatively. 


\section{TABLE 1. Operative data}

\begin{tabular}{lc}
\hline Patient characteristics & \\
No. of patients & 35 \\
Men/women & $13 / 22$ \\
Age, y (mean) & $47-80(67.8)$ \\
Operative data & \\
Operative time, min & $338 \pm 86$ \\
Cardiopulmonary bypass time, min & $175 \pm 41$ \\
Selective cerebral perfusion time, min & $82 \pm 20$ \\
Diameter of stent graft, mm (mean) & $22-30(26.4)$ \\
Length of stent graft, cm (mean) & $7-12(8.8)$ \\
\hline
\end{tabular}
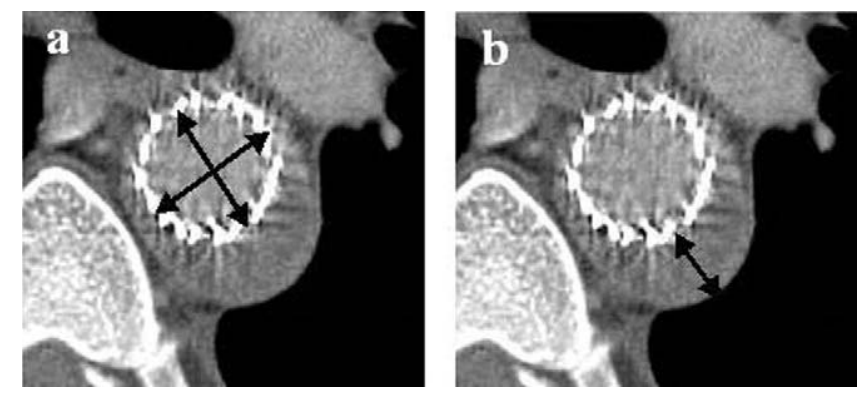

Figure 2. Measurement of the luminal diameter. A, The diameter of the true lumen was measured in 2 directions (long axis and short axis), and the mean value was calculated. B, The diameter of the false lumen was only measured along the short axis.

circulation time, and mean operating time were $82 \pm 20,175 \pm$ 41 , and $338 \pm 86$ minutes, respectively (Table 1$)$.

\section{Follow-up CT Study}

Two patients did not have dissection extending to involve the abdominal aorta, and 2 other patients died within 30 days after the operation. The other 31 patients were all followed up for at least 36 months until April 2005. The mean follow-up period was 61 months (range, 36-89 months). CT was performed at 1, 3, 12, and 36 months postoperatively to detect thrombosis and absorption of the false lumen that had been excluded by the stent graft (Figure 1). The diameter of the true lumen was measured in 2 directions (the maximal diameter and the minimal one), and the mean value was calculated because the true lumen is frequently egg shaped soon after the operation. The diameter of the false lumen was only measured along the short axis (Figure 2). Measurement was done at 3 levels: the distal edge of the stent graft, the diaphragmatic level, and the origin of the superior mesenteric artery (SMA; Figure 3).

\section{Results}

Table 1 summarizes the operative data. The mean diameter of the stent graft was $26.2 \mathrm{~mm}$ (range, 22-30 $\mathrm{mm}$ ), and the mean length was $8.9 \mathrm{~cm}$ (range, $7-12 \mathrm{~cm}$ ). Table 2 shows the early and late postoperative results. Two patients died within 30 days after the operation from low output syndrome and bleeding, respectively. However, paraplegia or intestinal ischemia did not occur in any of the patients. Late results were obtained for the other 31 patients after a follow-up period of 61 months (range, 36-89 months). None of the patients died or required additional surgical treatment of the thoracoabdominal aorta after discharge.

\section{Thrombosis and Obliteration of the False Lumen Thrombus formation in the false lumen was recognized in all patients at the distal edge of the stent graft, whereas it was seen in $26(84 \%)$ patients at the diaphragmatic level and in $15(48 \%)$ patients at the SMA level. Moreover, obliter- ation of the false lumen was recognized in all patients at the distal edge of the graft, whereas it was seen in $20(65 \%)$ patients at the diaphragmatic level and in 15 (48\%) patients at the SMA level (Table 3).}

\section{Diameter at the Stent Graft Edge}

The diameter of the false lumen decreased from 1 month postoperatively. It was less than $2 \mathrm{~mm}$ after 3 months in 22 ( $71 \%$ ) patients, and it was less than $2 \mathrm{~mm}$ by 1 year after the operation in all of the patients. The diameter of the false lumen also decreased from 1 month postoperatively, being less than $2 \mathrm{~mm}$ at 3 months in $22(71 \%)$ patients and less than $2 \mathrm{~mm}$ by 1 year after the operation in all patients. The mean expansion of the stent graft from 1 to 36 months after insertion was only $1.16 \mathrm{~mm}$ (range, 0-4 mm).

\section{Diameter at the SMA Level}

All 16 patients with thrombosis in the false lumen showed luminal obliteration by 1 year after the operation, whereas the abdominal aorta was unchanged in the 15 patients with a residual false lumen. In those with a residual false lumen at the SMA level, the mean enlargement of the whole aorta at this level was only $0.375 \mathrm{~mm}$ (range, $0-2 \mathrm{~mm}$ ) from 1 to 36 months after the operation.

\section{Diameter at the Diaphragmatic Level}

In patients with or without obliteration of the false lumen at the diaphragmatic level, the mean enlargement of the whole aorta at this level from 1 to 36 months after the operation was only $0.5 \mathrm{~mm}$ (range, $0-2 \mathrm{~mm}$ ).

\section{Discussion}

\section{Residual False Lumen in the Descending Thoracic Aorta (Residual Tear)}

Acute type A aortic dissection is associated with a high risk of death and frequently requires emergency surgical intervention, and therefore conventional replacement with a synthetic graft is often limited to dissection from the ascending aorta to the aortic arch. Therefore a false lumen sometimes remains in the distal part of the descending thoracic aorta after the operation. It has been reported that the distal false lumen remains patent in $50 \%$ to $70 \%$ of patients after ascending aortic replacement and in $15 \%$ to $30 \%$ of patients 

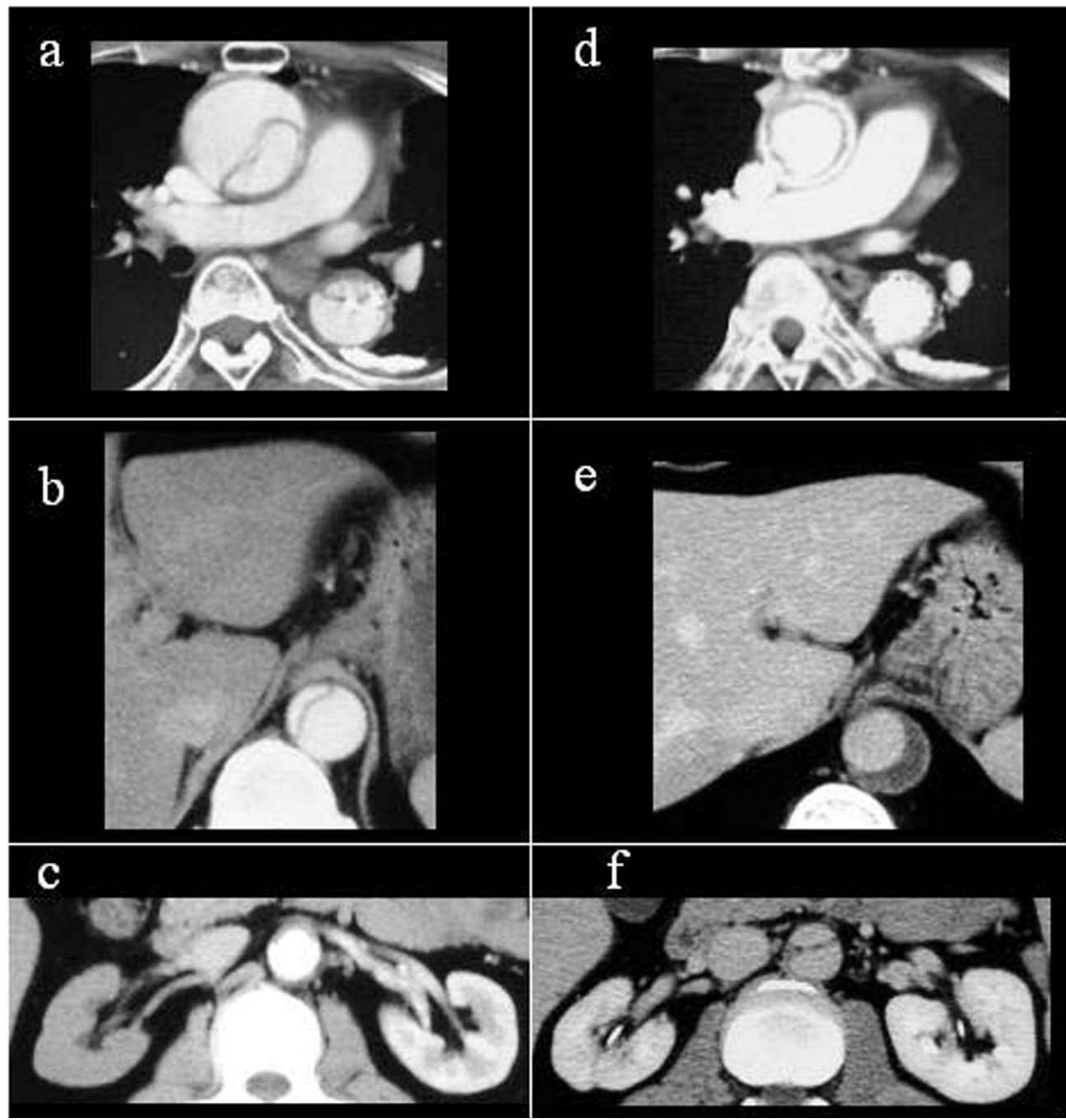

Figure 3. Sites of measurement. A, B, and C, Preoperative findings. D, E, and F, Three months postoperatively. A and $D$, Edge of the stent graft. $B$ and $E$, Diaphragmatic level. The narrow true lumen seen before the operation is dilated well after surgical intervention, and the false lumen shows complete thrombosis. C and F, SMA level. The occluded true lumen and right renal artery become patent after surgical intervention. A residual false lumen is seen in the abdominal aorta.

after aortic arch replacement. ${ }^{1,5-7}$ This lumen can significantly affect the early and late prognosis after surgical intervention. In the early postoperative period several of the patients treated for acute type A aortic dissection experience intestinal necrosis caused by poor perfusion of intra-abdominal branches of the aorta, and such malperfusion often occurs because the distal false lumen has remained patent. Moreover, reoperation is often required for dilatation of a 
TABLE 2. Postoperative data

\begin{tabular}{lc}
\hline Postoperative data & \\
Early results & 2 \\
Early mortality & 4 \\
Early morbidity & 0 \\
Cerebral accident & 0 \\
Paraplegia & 2 \\
Pulmonary failure & 1 \\
Renal failure & 1 \\
Low output syndrome & 0 \\
Intestinal ischemia & $30-83$ (55) \\
Late results & 0 \\
Follow-up period, mo (mean) & 1 \\
Late mortality & 1 \\
Late morbidity & 0 \\
Cerebral accident & 0 \\
Paraplegia & 1 \\
Pulmonary failure & 0 \\
Additional surgical procedure & 1 \\
Thoracic aorta & \\
Infrarenal abdominal aorta &
\end{tabular}

\section{TABLE 3. Findings on follow-up CT scanning}

\begin{tabular}{ll}
\hline Follow-up CT examination 36 mo after the operation \\
( $\mathrm{n}=31$ ) \\
Thrombosis of the false lumen \\
Distal edge of the graft \\
Diaphragmatic level & 31 \\
SMA level & 26 \\
Obliteration of the false lumen & 15 \\
Distal edge of the graft & 31 \\
Diaphragmatic level & 20 \\
SMA level & 15
\end{tabular}

$C T$, Computed tomography; SMA, superior mesenteric artery.

false lumen in the distal descending aorta during the late postoperative period. Because of anastomotic leakage or a small tear remaining in the proximal descending thoracic aorta after graft replacement of the ascending aorta or aortic arch, the false lumen is susceptible to dilation because of shear stress acting on the proximal descending thoracic aorta. Akutsu and associates ${ }^{8}$ have demonstrated that patency of the false lumen is a strong independent prognostic factor of dissection-related death and dissection-related events. Therefore there have been recent reports about total replacement of the aortic arch at the first operation in such patients, even if the primary intimal tear is located in the ascending aorta. Kazui and coworkers ${ }^{9}$ reported that repair of the descending thoracic aorta up to the midportion with a synthetic graft by using the elephant trunk technique at the initial operation could prevent subsequent dilation of the most susceptible parts of the residual false lumen. However, the diameter of a graft inserted by using the elephant trunk procedure is likely to be smaller than that of a distally stented graft. Use of a distally anchored stent graft allows insertion of a larger diameter and longer prosthesis than the elephant trunk method. ${ }^{10}$ When grafting is done with the elephant trunk procedure, the graft inserted is often smaller than that required in relation to the diameter of the true lumen, and therefore the elephant trunk method cannot completely prevent dilation of the residual false lumen. We determine the most appropriate size of the stent graft by measuring the diameter of the true lumen with a ball-shaped sizer during systemic circulatory arrest under transesophageal echocardiographic guidance. ${ }^{3}$ We often detect a small intimal tear using transesophageal echocardiography, ${ }^{11}$ insert the stent graft into the descending thoracic aorta at the level of the pulmonary artery trunk, and close the small tear with the inserted stent graft. The aim of this stent grafting technique is to exclude all antegrade blood flow in the false lumen of the thoracic aorta, therefore decreasing pressure and allowing thrombosis of the false channel to restore blood flow in the aorta and its side branches. In vitro models of dissection have shown that primary entry tear coverage is the optimal method to relieve true lumen collapse and false lumen thrombosis. ${ }^{12}$ Kato and colleagues ${ }^{4}$ reported that reoperation to treat the residual dissected aorta was only needed in 1 patient (1/17 [5.9\%]) after total arch grafting with open stent graft placement for acute type A aortic dissection. In the present series we had no patients who required additional surgical intervention of the thoracic aorta after this procedure. These results suggest that using a stent graft of the appropriate size and length, as determined by means of intraoperative measurement with a ball-shaped sizer inserted into the true lumen of the descending aorta from the transverse incision under transesophageal echocardiographic guidance, leads to a good outcome of open stent graft placement.

\section{New Intimal Tears}

Dilation of the true lumen of the descending thoracic aorta along with dilation of the false lumen during echocardiography-guided insertion of a self-expanding stent graft poses the risk of creating a new intimal tear. There have also been reports of new intimal tears in the late postoperative period after stent grafting. ${ }^{4,13,14}$ However, such new intimal tears almost correspond to chronic dissection. We have also reported a patient who required an additional operation 11 months after the first procedure because of ulceration caused by the stent graft. ${ }^{15}$ In patients with "chronic" dissection, the thrombosed false lumen persists in the early postoperative period. In addition, the true lumen is dilated at the central portion of the graft, which might increase turbulent flow through interaction with the stent. Such turbulence might damage the intima of the true lumen and lead to ulceration over the medium term postoperatively. However, this report is concerned with open stent grafting for "acute" 
dissection, and therefore the graft size determined by measuring the diameter of the true lumen during systemic circulatory arrest is the most appropriate, and moreover the intima of the true lumen suffers less stress in acute dissection than in chronic dissection. In our patients with acute dissection, the false lumen showed early thrombosis and early obliteration. This demonstrates that early stent grafting for acute dissection can maintain a normal diameter and blood flow through the true lumen and can completely exclude the false lumen. In addition, the true lumen is not dilated at the site of stenting, and therefore the stent will create less turbulence. ${ }^{4,15}$

\section{Residual False Lumen of the Thoracoabdominal Aorta} A residual false lumen of the thoracoabdominal aorta exists after an open stent grafting operation. However, when there was a residual false lumen or no obliteration of the false lumen below the diaphragm, the mean enlargement of the entire subdiaphragmatic aorta from 1 to 36 months postoperatively was only $0.5 \mathrm{~mm}$ (range, $0-2 \mathrm{~mm}$ ) and only 0.375 $\mathrm{mm}$ (range, 0-2 $\mathrm{mm}$ ) at the level of the SMA. Enlargement of the residual false lumen in the thoracoabdominal aorta is not recognized after complete closure of the primary tear once the false lumen in the descending thoracic aorta disappears after open stent grafting. All patients with a residual false lumen in the thoracoabdominal aorta have an intimal tear (so-called re-entry), and the blood flow through this tear supplies one or more of the main abdominal branches, such as the SMA, renal arteries, and celiac artery. Therefore the diameter of the false lumen in the thoracoabdominal aorta shows minimal changes after the operation because the false lumen is acting as a vessel that provides blood to the abdominal organs.

\section{Risk of Paraplegia}

The risk of paraplegia caused by sacrificing the spinal arteries must be considered when a long synthetic vascular graft is inserted into the descending thoracic aorta. In our experience the mean length of the stent graft was $8.9 \mathrm{~cm}$ (range, 7-12 cm), and therefore the graft was inserted as far as the Th9 level. Perfusion of all 3 branches of the aortic arch with a single balloon cannula and perfusion of the descending thoracic aorta with 2-way balloon cannulas under hypothermic conditions $\left(25^{\circ} \mathrm{C}\right)$ prevented spinal cord ischemia during open distal anastomosis with selective cerebral perfusion.

\section{Conclusions}

This open stent grafting procedure for acute type A dissection might be able to prevent type $\mathrm{B}$ dissection of a residual false lumen in the late postoperative period. Thus open stent grafting of the distal descending aorta with total arch replacement achieves extended reconstruction of the thoracic aorta from the ascending to distal descending aorta. This might reduce the need for a second operation not only for the distal descending aorta but also for the thoracoabdominal aorta.

\section{References}

1. Bachet J, Teodori G, Goudot B, Diaz F, el Kerdany AE, Dubois C, et al. Replacement of the transverse aortic arch during emergent operation for type A acute aortic dissection. J Thorac Cardiovasc Surg. 1988;96:878-86.

2. Ergin MA, Phillips RA, Galla JD, Lansman SL, Medelson DS, Quintana CS, et al. Significance of distal false lumen after type A dissection repair. Ann Thorac Surg. 1994;57:820-5.

3. Ishihara H, Uchida N, Yamasaki C, Sakashita M, Kanou M. Extensive primary repair of the thoracic aorta in Stanford type A acute aortic dissection by means of a synthetic vascular graft with a self-expandable stent. J Thorac Cardiovasc Surg. 2002;123:1035-40

4. Kato M, Kuratani T, Kaneko M, Kyo S, Ohnishi K. The results of total arch graft implantation with open stent-graft placement for type A aortic dissection. J Thorac Cardiovasc Surg. 2002;124:531-40.

5. Fann JI, Smith JA, Miller C, Mitchell S, Moore KA, Grunkenmeier G, et al. Surgical management of the aortic dissection during a 30-year period. Circulation. 1995;92(suppl II):II113-21.

6. Ando M, Nakashima N, Adachi S, Nakaya M, Kawashima Y. Simultaneous graft replacement of the ascending aorta and total aortic arch for type A aortic dissection. Ann Thorac Surg. 1994;57:669-76

7. Kazui T, Kimura N, Yamada O, Komatsu S. Total arch graft replacement in patients with acute type A aortic dissection. Ann Thorac Surg. 1994;58:1462-8.

8. Akutsu K, Nejima J, Kiuchi K, Sasaki K, Ochi M, Tanaka K, et al. Effects of the patent false lumen on the long-term outcome of the type B acute aortic dissection. Eur J Cardiothorac Surg. 2004;26: 359-66.

9. Kazui T, Tamiya Y, Tanaka T, Kamatsu S. Extended aortic replacement for acute type A dissection with the tear in the descending aorta. J Thorac Cardiovasc Surg. 2000;119:558-65.

10. Kato M, Matsuda T, Kaneko M. Outcomes of stent-graft treatment of false lumen in aortic dissection. Circulation. 1998;98(suppl II):II30512.

11. Orihashi K, Matsuura Y, Sueda T, Watari M, Okada K, Sugawara T, et al. Echocardiography-assisted surgery in transesophageal echocardiography. J Thorac Cardiovasc Surg. 2000;120:672-8.

12. Chung JW, Elkins C, Sakai T, Kato N, Vestring T, Semba CP, et al. True-lumen collapse in aortic dissection: part I. Evaluation of causative factors in phantoms with pulsatile flow. Radiology. 2000;214: 87-98.

13. Shimono T, Kato N, Yasuda F, Suzuki T, Yuasa U, Yada I, et al. Transluminal stent-graft placements for the treatments of acute onset and chronic aortic dissections. Circulation. 2002;106(suppl 1):I241-7.

14. Dake MD, Kato N, Mitchell RS, Semba CP, Razavi MK, Shimono T, et al. Endovascular stent-graft placement for the treatment of acute aortic dissection. N Engl J Med. 1999;340:1546-52.

15. Uchida N, Ishihara H, Sakashita M, Kanou M, Sumiyoshi T. Repair of the thoracic aorta by transaortic stent grafting (open stent). Ann Thorac Surg. 2002;73:444-9. 